\title{
La construction impérative de l'habitat durable
}

\author{
Origines et perspectives d'un mot d'ordre à venir
}

\author{
Sophie Némoz \\ (Cerlis, Université Paris Descartes - Sorbonne)
}

\begin{abstract}
Devant le caractère indiscutable du bien-fondé de l' "habitat durable », cet article revient sur les dynamiques sociales à l'origine de cette récente catégorisation des logements écologiques, pour rendre compte de son caractère construit mais aussi de sa dimension normative. En interrogeant ce concept d'habitat durable, l'objectif plus général est d'examiner celui de « développement durable » et les applications de ce dernier dans le secteur résidentiel. Ce faisant, nous mettons à jour un manque certain de considération pour l'habitable tel que les ménages l'envisagent aujourd'hui. Un tel enseignement permet d'éclairer la faible légitimité de cette nouvelle rhétorique voire idéologie - gouvernementale chez ses publics cibles. L'article procède en trois temps : d'abord nous examinons l'émergence, la mise en place du paradigme de l'éco-construction, ensuite nous faisons le point sur les stratégies, les redéfinitions de ce paradigme par ses différents acteurs, et enfin nous nous penchons brièvement sur le vécu de ce paradigme parmi les habitants.
\end{abstract}

Mots-clefs : développement durable, habitat durable, écologie, éco-construction

\section{Introduction}

La notion sociopolitique de « développement durable » date presque d'une trentaine d'années. Au fur et à mesure, le secteur résidentiel en est devenu l'un des domaines d'extension privilégiés. Désormais au cœur d'une actualité sans précédent, la nouvelle catégorisation, supranationale, de «logement durable» reste cependant encore largement à interroger. Cet essai se propose dès lors de retracer les dynamiques ayant œuvré à la construction de l'idée d'habitat durable et à son établissement en véritable mot d'ordre. ${ }^{1}$ La restitution de ce phénomène social dans ses trois dimensions - l'instauration de la notion d'habitat durable dans les sphères institutionnelle, marchande et familiale - révèle le système d'acteurs qui anime la montée en puissance de cette catégorisation. Les logiques d'agrégation autour de cette invention rhétorique sont encore peu démêlées. Il s'agit ici de les appréhender à un niveau assez fin pour pouvoir en observer les enchevêtrements et la complexité depuis les origines jusqu'aux perspectives contemporaines. Ainsi dans un premier temps nous examinons l'émergence, la mise en place du paradigme de l'éco-construction, ensuite nous faisons le point sur les stratégies, les redéfinitions de ce paradigme par ses différents acteurs, et enfin nous nous penchons brièvement sur le vécu de ce paradigme parmi les habitants

\footnotetext{
${ }^{1}$ La force de ce mot d'ordre, ses origines et les tensions qu'il suscite ont été examinées en profondeur dans notre recherche doctorale (NEMOZ 2009). C'est dans les coulisses de l'action publique que ce travail de thèse a été inauguré. L'enquête débute en effet au sein d'une institution (le Ministère de l'Ecologie, de l'Energie, du Développement Durable et de l'Aménagement du Territoire) et avec le soutien d'opérateurs (le PUCA et l'ADEME), directement impliqués dans l'élaboration des normes françaises afférentes à la durabilité des bâtiments. Pour faire apparaître ce qui fait réellement sens dans cette catégorisation récente des logements écologique, la recherche s'est efforcée de prendre du recul par rapport aux discours des autorités administrative en France, en se constituant tant à l'étranger (Finlande et Espagne) que sur des terrains locaux de projets français d'éco-construction, où les acteurs visaient à réaliser de l'habitat durable. Le caractère construit de la notion d'habitat durable a ainsi été analysé grâce aux témoignages d'une centaine d'entretiens biographiques avec les parties prenantes de ce mouvement de différenciation des biens immobiliers, que ce soit dans les sphères institutionnelle, marchande ou familiale.
} 


\section{1. Émergence. Origines du paradigme de l'éco-construction}

L'enjeu de cet article est donc tout d'abord de remonter aux sources d'inspiration de ce nouveau canon résidentiel. En empruntant une approche dite "généalogique » - c'est-à-dire visant à faire ressortir le «processus de gestation » à l'origine et au développement d'un paradigme de compréhension et d'action dans le monde (FLICHY 2001, p.53) - il est possible de relativiser le caractère nouveau de cette idée d'un bien-être domestique et d'une construction en adéquation avec la nature, sans pour autant sous-estimer les importantes variations de sa représentation.

\subsection{Fondements du paradigme de l'habitat durable}

Au regard de l'humanité, la création d'un cadre bâti en harmonie avec ses environs naturels est en effet une pratique originelle, une constante de l'histoire. En revanche, la rationalisation d'une telle préoccupation, en connaissance de cause environnementale, parait récente. Elle est le produit d'un long processus d'innovation incrémentale, d'une succession de petits changements. Chez les peuples primitifs, la résolution des problèmes entre les résidences humaines et leur milieu naturel procède d'une intuition vitale. Pour se mettre à l'abri des intempéries et des prédateurs, de nombreux groupes humains ont par le passé modifié la flore et la faune environnantes et ce, parfois jusqu’à leur détriment, tel le brulis pratiqué par les Aborigènes d'Australie (JOHNSON, 2006). Avec l'industrialisation des sociétés, il est attribué à la nature une fonction hygiéniste et récréative. En France, c'est notamment Le Corbusier qui reconsidère dans une perspective fonctionnaliste les interactions entre le logement et les caractéristiques biophysiques du site, en proposant un nouveau mode d'aménagement des habitations, résumé dans la Charte d'Athènes en quelques mots : «soleil-espace-verdure » (LE CORBUSIER, 1943). Grâce à ces trois composantes naturelles, l'architecte estime que le territoire urbain peut satisfaire les fonctions clefs " habiter, travailler, se récréer » (LE CORBusier, 1943). L'habitation de la nature n'a pas été l'objet d'une catégorisation linéaire. La consultation des archives la montre liée aux facultés cognitives et comportementales des hommes. Dans un premier temps, les manières de construire avec le biotope ont été transmises par un savoir préréflexif, c'est-à-dire une compétence aveugle à elle-même et dont l'habileté s'acquière à force de répétition des gestes séculaires. Plus tard, au cours du XIXe siècle, l'art de bâtir devient un savoir scientifique qui s'élabore par abstraction des observations (ASSEGOND, 2004). Au cours de cette transition épistémique, la notion d'écosystème est imaginée (TANSLEY, 1935).

Le mouvement se poursuit par la médiation de l'habitat écologique en affaire sociale, prenant place dans le contexte de l'urbanisation occidentale des années 1960-1970. Ce phénomène interculturel est porté par ce que Moscovici a appelé des «mouvements naturalistes » (MOSCOVICI 2002, p.42), c'est-à-dire des mobilisations œuvrant à la transformation des rapports à la nature. Les interactions humaines avec l'écosystème deviennent un sujet d'inquiétude résidentielle dans une configuration urbaine en croissance massive. Des zoologistes et des botanistes inculpent les villes des pays industrialisés de la dégradation des biotopes. La critique se propage chez les intellectuels de l'époque. The City in History, pour ne citer que cet ouvrage, est publié en 1961. L. Mumford y détruit le «mythe de la mégalopole » et pronostique son déclin au vu de ses effets pervers (1961). Les témoignages oraux, notamment ceux recueillis auprès des membres des premiers partis écologistes en Finlande et en Espagne, insistent également sur les conséquences non maitrisées, incertaines sur le plan environnemental comme social, de la nouvelle donne urbaine (NEMOZ 2009). En France, cette conscience environnementale s'est incarnée, entre autres, dans la variété des composantes de mai 68. Dans le flux des interactions transgressives, la dégradation des conditions naturelles d'habitation a été imputée à l'État. La planification des reconstructions résidentielles d'après-guerre figure en effet parmi les multiples situations de domination publique dénoncées pour leur oppression de la vie. Mais ce procès de publicisation des problèmes de cohabitation entre nature et société transcende les spécificités territoriales. S'il ne prend pas la même forme protestataire que sous les pavés parisiens, le phénomène atteint une envergure géopolitique au moment des chocs pétroliers (dès 1973). Les tensions autour de l'énergie fossile aiguisent l'écho des discours techno-apocalyptiques qui questionnent au niveau planétaire le perfectionnement des technologies, l'augmentation sans précédant de la population et l'essor inégal de l'économie, à la croisée de plusieurs discours philosophiques pourtant très divers mais portant tous sur une certaine défiance vis-à-vis de la technique (Jonas, Heidegger, Nietzsche, etc.). 
Ainsi, l'enjeu écologique des normes de construction monte en puissance. En 1976, il est reconnu lors d'un premier sommet international : la conférence des Nations Unies à Vancouver sur les établissements humains, dite aussi «Habitat I». Dans l'arène onusienne, les dommages environnementaux et socioéconomiques d'un monde de plus en plus urbain sont clairement pointés. La perspective de leur résolution au moyen de l'écologie résidentielle ne s'est pas conçue en un jour. Plutôt, elle s'est dessinée en quasiment deux décennies durant lesquelles un projet à l'origine marginal a été reformulé par l'expression d'« habitat durable ». La réinvention sémantique de l'éco-construction est en effet proclamée en 1996, à Istanbul, lors $\mathrm{du}$ «Sommet des villes » également nommé « Habitat II ». La formulation de cette catégorie se réfère explicitement à l'expression de " développement durable », dont le concept synthétise la prise en compte simultanée des interactions entre les dimensions économique, écologique et sociale des projets répondant à des questions de gestion de ressources ou d'aménagement. Bien qu'abstraite, la rhétorique guide l'imagination sur la voie des solutions de long terme, en appelant à la satisfaction des besoins présents, voire pressants en hébergements, ainsi que ceux des générations futures. Progressivement, l'écoconstruction est alors inscrite dans un projet de réduction des inégalités des conditions de vie (que ce soit dans le domaine de la santé, du confort, ou, en général, de l'accès aux ressources essentielles pour l'existence).

En étudiant comment la catégorie des logements écologiques s'est reconstruite à travers l'idée de durabilité, nous pouvons plus précisément reconstituer le «modèle séquentiel de légitimation d'une nouvelle technique » que P. Flichy a mis à jour, à partir du cas de l'innovation d'internet (2001, p.61). Cette analyse a l'avantage d'éclairer l'imaginaire techno-messianique que l'appellation de l'habitat durable confère au développement des techniques d'éco-construction. Ce phénomène caractéristique du processus d'une innovation permet en l'occurrence de surmonter l'antagonisme que l'écologisme a initialement dénoncé entre la croissance urbaine et la préservation de l'environnement naturel. En d'autres termes, ceux du sociologue P. Flichy, il est possible de remarquer la création d'une «idéologie-masque » autour des logements écologiques (2001, p.61). La formulation du développement durable ne reflète pas le détail des modalités techniques où les divergences entre pionniers de l'éco-construction se cristallisent. Autrement dit, il s'agit d'un terme trompe-l'œil, regroupant une pluralité dans une totalité d'apparence cohérente. Sous l'égide de G. H. Brundtland, tout se passe comme si la Commission Mondiale de l'ONU sur l'Environnement et le Développement cherchait à concilier deux enjeux alors bien souvent opposés dans les discours écologistes. Leurs arguments sont modérés par une perspective de changements technologiques aux profits environnementaux. "Notre avenir à tous » est ainsi présenté dans un programme assez flou en 1987 (CMED), mais proche de ce que M. Janicke nomme « la modernisation écologique » (2008).

La rationalisation environnementale de l'hébergement humain gagne en visibilité à partir du Sommet de la Terre (Rio, 1992), où le développement durable s'expose à de nombreux chefs d'États, comme un raisonnement annonçant des doubles bénéfices pour l'économie et l'environnement dans certains domaines, et notamment dans le secteur résidentiel. Pour les techniques de construction écologique, une nouvelle séquence commence, correspondant à celle que P. Flichy nomme l'« idéologie-légitimante » (2001, p.62). Les tenants de l'ordre supranational les valorisent au point de prôner l'abandon de tout autre type de construction résidentielle. En effet, l'édification raisonnée selon des impératifs a) de précaution en matière d'environnement, b) de pollueur-payeur, c) de responsabilité internationale, et d) de participation citoyenne ${ }^{2}$, est officiellement promue comme la politique à mettre en œuvre sur une planète en constante urbanisation. De là, une mise en demeure s'affirme. Un « Agenda 21 local » est rédigé à l'attention des collectivités territoriales en vue d'une structuration plus cohérente du développement métropolitain, notamment en contenant l'étalement sur-consommateur de foncier et d'énergie. Un « Agenda 21 industriel » est également spécifié par l'Organisation mondiale de normalisation (ISO), mettant en avant des méthodes d'analyse écologique des produits de construction. Ces dernières s'adressent aux entreprises et certifient leurs performances environnementales pour mieux informer les habitants. Au seuil du XXIe siècle, appa-

\footnotetext{
${ }^{2}$ Cette série de principes proclamés par la Déclaration de Rio sur l'environnement et le développement, a été réaffirmée dans le Programme pour l'habitat, texte adopté par 171 gouvernements en 1996, et actuellement disponible à l'adresse : http://ww2.unhabitat.org/unchs/french/hagendaf/index.htm
} 
rait ainsi une «idéologie-mobilisation", c'est-à-dire une idéologie dont "la fonction [est] de mobiliser les producteurs de la technologie comme ses usagers » (FLICHY 2001, p.62).

\subsection{Différents acteurs, différentes implications}

Dans un contexte où l'économie remodèle les fonctions et les frontières physiques des États, la mondialisation des marchés du bâtiment fait apparaitre le rôle déterminant que de nouveaux acteurs conquièrent avec cette légitimation de l'habitat durable. Elle invoque des opérations saines et confortables pour tout un chacun qui préservent l'ensemble des éléments naturels : la faune, la flore, la terre, l'eau, l'air et les ressources énergétiques ainsi que le patrimoine historique et naturel, grâce à des moyens de production peu coûteux en transport. Le flou ainsi engendré de la notion englobante d'habitat durable possède cependant une utilité : celle de laisser à des acteurs aux compétences et traditions différentes une latitude pour en éclaircir et implémenter les composantes pratiques, en fonction de leurs enjeux.

L'Union Européenne mettra ainsi plus particulièrement l'accent sur les performances économes en énergie fossile (directive 2002/91/CE), de manière à consolider l'autonomie supranationale en la matière et à honorer ses prétentions fortes quant à la lutte contre le réchauffement climatique. Le délai de transposition dans les États membres est fixé au 4 janvier 2006, alors qu'il n'existe aucun mandat communautaire pour la politique résidentielle. Cet événement reflète une certaine transformation de l'action publique dans le secteur. Les récits finnois, hispaniques et français collectés dans notre thèse (NEMOZ 2009) convergent pour décrire le processus comme l'institutionnalisation d'un nouveau «monde d'objets », en faisant référence à « un ensemble de dispositifs extrêmement divers qui participent à une totalité d'appartenance » (BARBIER 2005).

En langue finlandaise, l'habitat durable renvoie à la poursuite des efforts d'adaptation à un environnement rude mais, adopté par des constructions bien isolées du froid avec les matériaux renouvelables issus du bois. Le bénéfice écologique de cet art de bâtir se réduit, voire vire au négatif par l'ajustement des parcours résidentiels vers des terrains constructibles en pleine forêt. Ceux-ci se situent bien souvent à plusieurs kilomètres des villes, parcourus quotidiennement en transport automobile.

Tandis que le gouvernement finnois tente d'inverser le rapport entre un enjeu global et des contraintes locales, les Communautés Autonomes concèdent une partie de leurs pouvoirs en tant qu'autorités régionales, dans une restructuration moins décentralisée des normes environnementales. Le choix de cette réorganisation sur la base d'un nouveau code national de la construction vise à réguler l'explosion urbaine par une législation énergétique en conformité avec les ambitions de l'UE. Ce faisant, l'injonction au développement durable soumet la souveraineté des États à des tensions à la fois externes et internes.

Enfin, côté français, le renforcement des instruments de contrôle des logements écologiques s'avère piloté par les grandes corporations de fonctionnaires. Ce sont les ingénieurs des Ponts et chaussées et de l'École des mines qui ont adapté le référentiel de l'habitat durable pour le redéfinir en une marque nationale d'éco-construction. Le but initial était de contrer les critiques des militants soixante-huitards à l'encontre de la politique d'industrialisation du bâtiment, et de conforter l'État dans son rôle d'entrepreneur. Le domaine ne cesse aujourd'hui d'être porté par les élites administratives, sachant désormais que l'idée de durabilité s'énonce comme un véritable mot d'ordre international pour les décennies à venir. Sa consécration en France s'est opérée avec le Grenelle de l'Environnement, dont les objectifs s'étendent à 2020, voire 2050. Ce cycle de réunions organisées par le ministère en charge du secteur et avec les différents corps de métiers du bâtiment, est à l'image de l'institutionnalisation nationale de l'écologie résidentielle. L'événement est initié par le haut de la société, où s'établit le standard commercial de la qualité environnementale des résidences. Dès lors, l'effet de cette catégorisation technocratique de l'habitat durable sur la mise en œuvre bâtie ne peut être présumé, avant de se rendre sur les terrains locaux de l'éco-construction. 


\section{Stratégies. La reconfiguration du paradigme de l'éco-construction par ses acteurs}

Le logement ne se cantonne pas à un champ de l'action publique. Il renvoie bien sûr également à un édifice matériel et local, produit par un milieu professionnel. A écouter les personnes actives sur les chantiers français d'éco-construction ${ }^{3}$, ceux-ci s'inscrivent complètement dans le paradigme du développement durable. Dorénavant, les principes de ce dernier font figure de boussole pour la croissance verte. Vingt ans après la thèse de S. Juan sur l'édification de maisons solaires en France (1985), le paysage socioprofessionnel de l'éco-construction s'est considérablement diversifié. Notre recherche repère que les constructions de logements écologiques ne sont plus seulement l'œuvre de militants en dissidence avec l'État (NEMOZ 2009). Elles se propagent vers d'autres milieux sociaux si bien que de nouvelles formes de résistances et de coopérations collectives se manifestent vis-à-vis des instruments de la politique nationale de développement de l'habitat durable. De fait, comme le sociologue N. Alter l'a souligné, «l'innovation (...) c'est l'histoire d'un état de tension permanente entre les possibilités que représente l'invention et les choix collectifs qui en sont progressivement tirés » (2003, p.13).

Pour les analyser, le concept de «système d'action concret » peut être emprunté à l'approche stratégique de la sociologie des organisations. Elle désigne ainsi « l'institution toujours précaire et problématique d'une structuration humaine des champs de l'action, d'un mode de contrôle social gouvernant l'action collective des hommes dans une sphère d'emprise donnée » (CROZIER \& FrIEDBERG 1977, p.282). En ce qui concerne l'habitat durable, le degré d'acceptation des changements nécessaires au passage vers un nouveau système caractérisé par l'idée de viabilité des espaces bâtis, dépend de la propension des différents acteurs à identifier la promesse d'une évolution conforme à leurs besoins. Les stratégies ${ }^{4}$ adoptées à l'égard de la production écologique des habitations sont plus concrètement tributaires des opportunités et des contraintes données par le mouvement normatif de l'État vers le secteur. On observe ainsi trois types de stratégies collectives, de jeux d'acteurs qui se réclament de l'habitat durable : tout d'abord celui, à la campagne, des « francs-tireurs » de l'éco-construction, deuxièmement celui des pouvoirs publics, et troisièmement celui des industries des maisons préfabriquées en France. Dans chaque cas, les interprétations du mot d'ordre public en faveur de l'habitat durable ne correspondent pas, mais pour différentes raisons, à celle imaginée à la tête politico-administrative du pays.

C'est donc à la campagne que le premier jeu d'acteurs de l'éco-construction française a pris forme. Un aspect transgressif ressort de ces pratiques locales. Bien après les communautés soixante-huitardes, des ménages issus des classes moyennes manifestent de l'intérêt pour un style de vie néo-rural, poursuivant à l'heure de l'institutionnalisation des principes de durabilité la séquence de diffusion des logements écologiques que les pionniers avaient initiée dans les années 1960-1970. Des particuliers échafaudent des projets de résidences individuelles qui reprennent les techniques ancestrales de construction, à base de matériaux naturels, et ce à l'instar des anciennes minorités écologistes. Car s'il y a là un phénomène d'imitation, il a cependant lieu sans référence directe aux convictions des initiateurs. Ainsi que le sociologue J. Ion et ses confrères l'ont souligné, les «militants d'aujourd'hui» se différencient nettement de ceux d'hier, en cela que leurs actions sont moins motivées par des valeurs que par la perspective d'« apporter une réponse immédiate, même provisoire, aux problèmes considérés » (ION et al. 2005, p.7). Ce changement comportemental nous a été confirmé, lors de notre thèse (NEMOZ 2009), par des familles qui ont investi le rôle de maitre d'ouvrage écologique pour leur propre compte. Précisément, leurs propos relatent les crises polymorphiques qu'elles ont vécues en ville : sur fond de désagréments urbains éprouvés au quotidien, des événements marquant leur cycle de vie privée (cancer d'un jeune enfant, départ des plus âgés pour des études supérieures loin du foyer parental, arrivée d'un nouveau-né, ou encore l'héritage d’une vieille ferme) ont déclenché leurs décisions de jouer les «francs-tireurs » de l'habitat durable. Cette expression, reprise à l'approche interactionniste d'Howard Becker, définit une catégorie d'individus qui ne s'en tiennent pas aux

\footnotetext{
${ }^{3}$ Pour ces entretiens, lire NEMOZ 2009

${ }^{4}$ Par «stratégies », sont entendues des rationalités limitées, plurimodales (instrumentales, axiologiques, cognitives, institutionnelles) qui s'exercent en fonction des situations et des relations de pouvoir, les « jeux » se formant dans l'interaction concrète de ces logiques d'acteurs (CROZIER \& FrIEDBERG 1977, p.97)
} 
règles consacrées par le milieu pour réaliser leurs œuvres et qui, en conséquence, apparaissent " déviants » au regard des "professionnels intégrés », que ce soient les juges, les représentants ou les producteurs du style conventionnel (BECKER 1988, pp.250-252). C'est un regard discriminant qui s'exerce sur la conception et l'édification de maisons bucoliques où la notion de durabilité s'exécute par l'art de bâtir avec des matières extraites de la nature locale, à l'image du patrimoine construit durant des générations. Concrètement, la contrainte qui pèse sur ce premier jeu d'acteurs de l'éco-construction est de nature procédurière. En effet, « la mise en œuvre du chanvre, de la ouate de cellulose, des isolants à base végétale (bois et autres fibres), ou à base animale (plumes de canards, poils de porcs, laines de moutons, etc.), suscite aujourd'hui des réticences à assurer la garantie décennale » (SOPRIANO 2007, p.63). Etant donné que cette garantie est obligatoire pour tous les corps de métiers du bâtiment, depuis l'adoption par l'État français de la loi dite «Spinetta » en 1978, la frilosité du milieu assurantiel n'est pas une variable négligeable pour l'innovation du secteur. Cette expérience s'avère chaotique, faute de police d'assurance adaptée. Ainsi, ce jeu d'acteurs se place entre la liberté de créer de nouvelles activités constructives et la précarité d'une position sans normes statutaires. À la veille du Grenelle de l'Environnement, tenu en France en 2007, cette situation de tension avait d'ailleurs été mise en avant par les porte-paroles de ce mouvement. L'enjeu était de défendre l'expertise en habitat durable de ces métiers en reconversion, afin d'améliorer leur encadrement politicoadministratif. Celui-ci ne régule pas encore les aspirations à une meilleure qualité de travail des professionnels qui, dans le cadre de projets de constructions plus traditionnelles, souffrent d'une très faible valorisation des travailleurs (JOUNIN 2008). Il n'empêche que des transformations se produisent dans les métiers mobilisés par l'éco-construction (nouveaux métiers, transformations des métiers traditionnels...).

On voit ainsi apparaitre le deuxième jeu d'acteurs et ses stratégies : celles des pouvoirs publics, dont certaines caractéristiques ont déjà été abordées plus haut. Ceux-ci ont investi différemment la dimension sociale de l'habitat durable, en proposant des programmes expérimentaux à toutes les catégories socioéconomiques des ménages, des familles aisées aux plus démunies. Ils ont pour effet un jeu stimulant l'action des groupes, initialement moins impliqués. Ce deuxième phénomène organisationnel dans la production actuelle de logements écologiques instaure divers rattachements institutionnels, que ce soit via des appels d'offre «SUNRISE » (subventionnés par l'Union Européenne, qui cherche ainsi à inciter l'usage de l'énergie solaire dans l'habitat) ou bien via des projets d'expérimentation résidentielle du ministère français en charge de l'infrastructure territoriale (les "Villas Urbaines Durables»). Nous pouvons aussi déceler chez les municipalités qui accordent le permis de construire à de tels projets résidentiels, le besoin de superviser leur peuplement. La formule de l'habitat durable est associée, à divers niveaux, à l'idée de légitimités inédites et à l'incertitude du contenu des transformations prévues par l'État. S'il s'est séparé de certaines fonctions décentralisées aux collectivités locales, ou intégrées à l'espace communautaire des politiques publiques, l'importance de la présence étatique n'est pas à sous-estimer. Dans le cadre de concours d'architecture expérimentale et canonique de la nouvelle politique du logement, les architectes et, plus largement, les professionnels de la construction sont conscients de construire leur position au sein de leur hiérarchie professionnelle. Pour la maitrise d'ouvrage, il en va de la recherche d'une meilleure gestion des nouvelles exigences sectorielles; les petites et moyennes entreprises du bâtiment, en changement d'activités, sont en quête de chantiers démonstratifs de leur valeur ajoutée. Quant aux bureaux d'études, leur désir de prouesses technologiques est palpable.

Ces cadres posés par les acteurs étatiques influencent en outre un troisième jeu d'acteurs de l'habitat durable : les industriels des maisons préfabriquées en France. En effet, dans le cahier des charges de la marque nationale d'éco-construction: «HQE® $» 5$, il y a des instructions qui préfigurent un jeu de production standardisée et décrite comme une voie de développement durable pour le secteur résidentiel. Pourtant, aucun changement radical n'est constaté au niveau des procédés de fabrication. C'est une logique additive où les modes de construction de l'entreprise sont pour l'essentiel maintenus. La prise en compte de la donne environnementale se limite à des technologies en bout de chaîne dans cette troisième collaboration d'acteurs. L'installation de ces technologies vertes est proposée sous forme d'options applicables aux anciennes gammes de résidences pavillonnaires. En ce sens, elle pousse à l'extrême un processus largement

\footnotetext{
${ }^{5}$ Haute Qualité Environnementale.
} 
incrémental d'innovation résidentielle. On peut presque parler d'une dilution de l'éco-construction dont les principes actifs deviennent plus édulcorés. Elle est aussi filtrée socialement par les ménages occupants.

\section{Vécu. Eco-construction et attitude pragmatique des habitants}

Ayant passé en revue les principales caractéristiques de l'émergence du paradigme de l'habitat durable d'abord, et des stratégies et jeux d'acteurs qui s'y retrouvent ensuite, il s'agit maintenant de brièvement faire le point sur son vécu. Autrement dit, il nous faut examiner le point de vue des habitants vivant dans un habitat durable. Il ressort que dans le présent contexte de crise quantitative (pénurie et cherté de l'offre) et qualitative du logement (inadaptation de l'offre à une multiplicité de situations domestiques), l'emménagement dans un bâtiment distinct par sa qualité environnementale dépend in fine des problèmes qu'il peut résoudre au-delà de sa fonction écologique. De fait, les habitants, même militants, ne l'envisagent pas uniquement dans une logique axiologique (du point de vue des valeurs éthiques) - et, plus généralement, le rôle des valeurs ne parait guère le plus décisif des processus amenant les divers milieux sociaux à ce changement résidentiel. On assiste actuellement à une appropriation ambivalente des cadres idéologiques liés à l'habitat durable. La représentation que les ménages se font de l'habitat durable est contrainte par l'ensemble des interprétations possibles de cette catégorie sociopolitique qu'autorise, par différent biais, la société dans laquelle s'inscrivent ces familles. La cohabitation harmonieuse avec l'environnement est à la fois un rapport individuel (sensation du rapport physique à la nature) et collectif (préservation par une action coordonnée et territorialisée). A partir des catégories propres aux habitants, il est important de rappeler que la qualité de vie recouvre deux niveaux différents. Ceux-ci méritent d'être distingués parce qu'entre celui des besoins et celui du bien-être, il n'y a pas le même caractère d'urgence. Les conditions de vie renvoient à la satisfaction des besoins élémentaires nécessaires à la survie et à la dignité humaine. Si le bien-être pose ceci comme préalable, il intègre en plus l'idée d'aspirations et de perceptions.

Alors que cette dernière motive des ménages citadins à se réfugier à la campagne, ces habitants néoruraux se heurtent à une territorialité critique. En refusant de choisir entre la quiétude d'une maison dans la prairie et l'hyperactivité d'une ville à plusieurs kilomètres de là, les principaux concernés admettent le paradoxe de leur situation résidentielle par rapport à l'écologie. Sur le plan individuel, ils éprouvent une forme de désordre qui renvoie à la cognition incohérente de deux éléments et ce, telle que L. Festinger conçoit la «dissonance cognitive» (1957). Le phénomène se produit plus précisément lors des déplacements motorisés que les ménages jugent tout autant nocifs pour l'environnement, que vitaux pour les liens sociaux. En d'autres termes, ceux de P. Bourdieu, la «distinction» est de mise sur le plan social au sens où, comme ce sociologue l'explique, "pour être reconnu dans un champ il faut s'y distinguer tout en évitant d'en être écart» (1979).

Plus que la différenciation sociale, c'est davantage d'équité qui est recherché au niveau des principes internationaux de l'habitat durable. A leur encontre, les signaux envoyés par les marchés locaux sont contradictoires. L'offre de terrains constructibles en zone rurale est accessible aux divers niveaux de revenus des ménages issus des classes moyennes. Cela est de moins en moins le cas en ville, compte tenu des prix du foncier. Toutefois, à la campagne, une hausse du budget consacré aux déplacements journaliers est à prévoir, avant même de verser le premier acompte pour le devis des travaux. En effet, la flambée des prix du pétrole, dont beaucoup d'éléments font penser qu'elle restera forte dans les prochaines années, menace les ménages néo-ruraux de précarité énergétique. Il s'agit d’un risque qui pèse sur la justice sociale. En conséquence, cette interprétation de l'habitat durable en forme de valorisation du patrimoine rural peut paraitre quelque peu dévoyée par rapports aux objectifs officiellement désignés par la formule. Des parcelles de nature champêtre sont obérées et les écarts spatiaux se creusent entre les catégories sociales, à défaut de renforcer leur mixité.

Celle-ci s'observe en revanche dans le cadre légitime et médiatique des programmes publics de logements écologiques. Véritables vitrines des politiques de l'habitat durable, les habitants sont régulièrement exposés à tout un tas de questions. La gêne occasionnée a été exprimée de diverses manières au sociologue, menant son enquête de porte-à-porte. Il y les portes qui restent fermées, et celles qui s'ouvrent à la confidence de l'envers du décor, si l'on peut dire, puisque les foyers rencontrés laissent voir le manque de 
prise sur des technologies domestiques encore peu familières à l'usage, et dont l'apprentissage s'est d'après eux, limité à la connaissance d'une liste d'interdits à l'égard de tout réaménagement spatial. Si c'est un regard subjectif sur la politique de développement de l'habitat durable, vue d'en bas, il rejoint un fait moins contestable mais, tout aussi perturbant : la faible acquisition par les ménages primo-accédants.

Rencontrée dans les villages d'exposition des maisons de marque «HQE », cette catégorie de ménages visite les pavillons sans se projeter dedans, ni véritablement les associer à la recherche de leur première propriété. Leur méfiance est argumentée lorsqu'on écoute l'importance du statut de propriétaire dans l'ascension sociale de ceux qui ont de petits moyens, aujourd'hui insuffisants pour prétendre à une construction de «Haute Qualité Environnementale», une fois payée le terrain foncier. L'enjeu est donc de trouver une légitimité à agir pour la protection de l'écosystème qui articule davantage une réflexion sur l'habitable aujourd'hui. Compte tenu des lacunes encore existantes dans ce domaine de recherche, la conclusion vise à souligner la nécessité de se pencher davantage sur la pensée des habitants, les sensibilités et les rationalités qui influencent leurs modes d'habiter.

\section{Conclusion}

En retraçant les dynamiques institutionnelles de la catégorisation récente de l'habitat durable, celle-ci a pu être analysée comme l'idéologique techno-messianique des constructions écologiques. Sa concrétisation en de nouveaux biens immobiliers réputés plus "verts» ne va pas nécessairement remporter un grand succès auprès des ménages fragilisés. Il y a d'autres nécessités qui leur paraissent plus urgentes à réaliser que les impératifs politiques en faveur de l'habitat durable. Son caractère abstrait, véhiculé par des experts, reste encore très éloigné des préoccupations quotidiennes des familles de manière générale, même pour les plus motivées d'entre elles. Dans les campagnes françaises, les foyers militants expriment leur désir d'un bien-être avec la nature, un contact plus sensible mais aussi, plus lourd de conséquences pour l'écosystème local. La perspective d'une croissance laisse ainsi entrevoir une complexification des pressions entre environnement et société de consommation. S'il ne suffit pas d'introduire une série des principes internationaux pour que les consommateurs s'en emparent, les fassent leurs et les adoptent, engendrant alors les changements attendus, l'habitabilité urbaine est à régénérer plus largement, sans quoi il est à craindre un essoufflement local de la formule de l'habitat durable.

\section{Bibliographie}

ASSEGOND C. (2004): «Repenser la technique en sociologie du travail : ancien paradigme, nouvelles perspectives ». In Revue de l'IRES, n4 «Activité et action dans le travail», pp.171-188.

BARBIER R. (2005): Le monde du résidu. Pour une sociologie politique des objets. Metz: Université Paul Verlaine, Thèse d'habilitation à diriger des recherches.

BECKER H. (1988): Les mondes de l'art. Paris: Flammarion.

BOURDIEU P. (1979): La distinction. Critique sociale du jugement. Paris: Editions de Minuit.

Commission Mondiale sur L'Environnement et LE Developpement - CMED (1987): Our common future. Montréal: Editions du Fleuve.

Crozier M., Friedberg E. (1977): L'acteur et le système. Paris: Seuil.

FESTINGER L. (1957): A theory of cognitive dissonance. Standford: Standford University Press.

FLICHY P. (2001): « La place de l’imaginaire dans l'action technique ». In Réseaux, n¹09, pp.59-73.

FrIEDBerg E. (1993): Le pouvoir et la règle : dynamique de l'action organisée. Paris: Seuil.

ION J., FRANGUIDAKIS S., VIOT P. (2005): Militer aujourd'bui. Paris: Autrement. 
JANICKE M. (2008): «Ecological modernisation : new perspectives ». In Journal of Cleaner Production, 16, pp.557-565.

JoHnson C. (2006): Australia's Mammal Extinctions. 50,000-Year History. North Queensland: James Cook University.

JOUNIN N. (2008): Chantier interdit au public. Enquête parmi les travailleurs du bâtiment. Paris : La Découverte, coll. «Textes à l'appui / enquête de terrain ».

JUAN S. (1985): De la maison solaire à l'action anti-technocratique; une approche sociologique de l'écologisme en France. Paris: Université Paris VII Jussieu, Thèse de doctorat en Sociologie.

LATOUCHE S. (2010): Le pari de la décroissance. Paris: Fayard - Pluriel.

LE CORBusier (1943): La charte d'Athènes. Paris: Plon.

MUMFORD L. (1961): The City in History. San Diego: Harcourt Inc.

NEMOZ S. (2009): L'«éco-logis », une innovation durable... Analyse sociologique de l'écologie résidentielle en France et au détour de la Finlande et de l'Espagne. Paris: Université Paris Descartes - Sorbonne, Thèse de doctorat en Sociologie.

SOPRIANO V. (2007): Etude sur le développement de produits assurantiels permettant la diminution des gaz à effet de serre. Paris: Ecole Nationale des Ponts et Chaussées, Thèse de doctorat professionnel.

TANSLEY A. G. (1935): «The use and abuse of vegetational concepts and terms », In Ecology, n¹6, pp.284-307. 